\title{
Primaquine Phosphate
}

National Cancer Institute

\section{Source}

National Cancer Institute. Primaquine Phosphate. NCI Thesaurus. Code C771.

The phosphate salt form of primaquine, a synthetic, 8-aminoquinoline derivative with antimalarial properties. Although its mechanism of action is unclear, primaquine bind to and alter the properties of protozoal DNA. This agent eliminates tissue (exo-erythrocytic) malarial infection, preventing the development of the erythrocytic forms of the parasite which are responsible for relapses in Plasmodium vivax and ovale malaria. Primaquine is active against late hepatic stages (hypnozoites, schizonts). 